\title{
Capabilities and Performance of the High-Energy Energetic-Particles Instrument for the Parker Solar Probe Mission
}

M. E. Wiedenbeck ${ }^{* a}$, N. G. Angold ${ }^{b}$, B. Birdwell ${ }^{b}$, J. A. Burnham ${ }^{c}$, E. R. Christian ${ }^{d}$, C. M. S. Cohen ${ }^{c}$, W. R. Cook $^{c}$, R. M. Crabill ${ }^{c}$, A. C. Cummings ${ }^{c}$, A. J. Davis ${ }^{c}$, G. Dirks ${ }^{b}$, D. H. Do ${ }^{e}$, D. T. Everett ${ }^{b}$, P. A. Goodwin ${ }^{d}$, J. J. Hanley ${ }^{b}$, L. Hernandez ${ }^{c}$, B. Kecman ${ }^{c}$, J. Klemic $c$, A. W. Labrador ${ }^{c}$, R. A. Leske ${ }^{c}$, S. Lopez ${ }^{c}$, J. T. Link ${ }^{d}$, D. J. McComas ${ }^{f}$, R. A. Mewaldt ${ }^{c}$, H. Miyasaka ${ }^{c}$, B. W. Nahory ${ }^{d}$, J. S. Rankin ${ }^{c}$, G. Riggans ${ }^{c}$, B. Rodriguez ${ }^{b}$, M. D. Rusert ${ }^{c}$, S. A. Shuman ${ }^{d}$, K. M. Simms ${ }^{d}$, E. C. Stone ${ }^{c}$, T. T. von Rosenvinge ${ }^{d}$, S. E. Weidner ${ }^{f}$, and M. L. White ${ }^{a}$

a Jet Propulsion Laboratory, California Institute of Technology, Pasadena, CA 91109 USA

${ }^{b}$ Southwest Research Institute, San Antonio, TX 78228 USA

${ }^{c}$ California Institute of Technology, Pasadena, CA 91125 USA

${ }^{d}$ NASA / Goddard Space Flight Center, Greenbelt, MD 20771 USA

${ }^{e}$ Applied Physic Laboratory, Johns Hopkins University, Laurel, MD 20723 USA

${ }^{f}$ Princeton University, Princeton, NJ 08544 USA

E-mail: mark.e.wiedenbeck@jpl.nasa.gov

\begin{abstract}
NASA's Parker Solar Probe (PSP) spacecraft (formerly Solar Probe Plus) is scheduled for launch in July 2018 with a planned heliocentric orbit that will carry it on a series of close passes by the Sun with perihelion distances that eventually will get below 10 solar radii. Among other in-situ and imaging sensors, the PSP payload includes the two-instrument "Integrated Science Investigation of the Sun" suite, which will make coordinated measurements of energetic ions and electrons. The high-energy instrument (EPI-Hi), operating in the MeV energy range, consists of three detector-telescopes using silicon solid-state sensors for measuring composition, energy spectra, angular distributions, and time structure in solar energetic particle events. The expected performance of this instrument has been studied using accelerator calibrations, radioactive-source tests, and simulations. We present the EPI-Hi measurement capabilities drawing on these calibration data and simulation results for illustrations.
\end{abstract}

35th International Cosmic Ray Conference - ICRC2017

10-20 July, 2017

Bexco, Busan, Korea

${ }^{*}$ Speaker. 


\section{Introduction}

Since the beginning of the space age, energetic particles accelerated close to the Sun have been measured in situ near Earth as well as at other locations in the heliosphere. Particles with energies up to several $\mathrm{MeV}$ are sometimes observed in energetic storm particle (ESP) events where the acceleration by a CME-driven shock continues all the way to $1 \mathrm{AU}$. However, the highest energies, sometimes approaching a $\mathrm{GeV} / \mathrm{nuc}$, are reached by particles accelerated within a few solar radii of the photosphere. When these events are measured near Earth, their characteristics have been altered by a variety of transport effects including adiabatic focusing, pitch-angle scattering on magnetic turbulence, velocity dispersion, drift in the large-scale magnetic field, reacceleration, and spreading due to the random walk of the heliospheric field. Deconvolving the characteristics of the accelerated particle population close to the Sun from these transport effects can introduce large uncertainties [1].

NASA's Parker Solar Probe (PSP) mission [2] is being developed to address long-standing problems related to the origins of the solar wind and of solar energetic particles by making in-situ measurements close to the Sun. The PSP spacecraft, which is currently being prepared for a launch window that opens on 31 July 2018, will use a sequence of gravity assists from close flybys of Venus to achieve a highly elliptical orbit having perihelion distances that will gradually be reduced from 35 solar radii in late 2018 to 9.8 solar radii in late 2024 . The design of the mission, the spacecraft, and the scientific instruments have been described in a series of papers written near the start of the development phase and published as Vol. 204 of Space Science Reviews.

Energetic particle measurements on PSP will be made by the two-instrument "Integrated Science Investigation of the Sun" suite [3], which consists of EPI-Lo, a time-of-flight versus energy spectrometer operating below $\sim 1 \mathrm{MeV} /$ nuc and EPI-Hi, a set of solid-state detector telescopes operating above this energy using the $d E / d x$ versus total energy technique. This paper discusses the design characteristics of EPI-Hi as well as its performance based on calibrations using accelerator beams, radioactive sources, and Monte Carlo simulations.

\section{Instrument Description}

Figure 1 shows a photograph of the EPI-Hi instrument shortly before it was delivered to the spacecraft team for integration in May 2017. The sensor system consists of three solid-state detector telescopes. The primary low-energy telescope, called LET1, has entrance apertures at both ends and is mounted on the spacecraft so that one end (called the "A-end") has a generally sunward view centered along a line $45^{\circ}$ west of the spacecraft-Sun line. Over much of the orbit, the nominal Parker-spiral magnetic field direction falls within this aperture. The other end ("B-end") of LET1 looks anti-sunward and will be able to see particles that are streaming inward, as occurs, for example, when they are reflected back by constrictions in the field out beyond the spacecraft. A secondary low-energy telescope, called LET2, is designed to be very similar to LET1 but omits the back aperture, which would be blocked by the spacecraft. LET2 is mounted to have a view orthogonal to the axis of the LET1 telescope. In this orientation, LET2 is well positioned to measure particles with pitch angles close to $90^{\circ}$ from the nominal field. A high-energy telescope, called HET, is designed to measure particles at higher energies than is possible with LET1 and 
LET2, while providing some energy-overlap with the LET measurements. Like LET1, HET is double ended, but its axis is closer to the spacecraft-Sun line $\left(20^{\circ}\right)$ than the axis of LET1. All three telescopes have their axes in the PSP orbit plane and have fields of view with half-angles of approximately $45^{\circ}$.

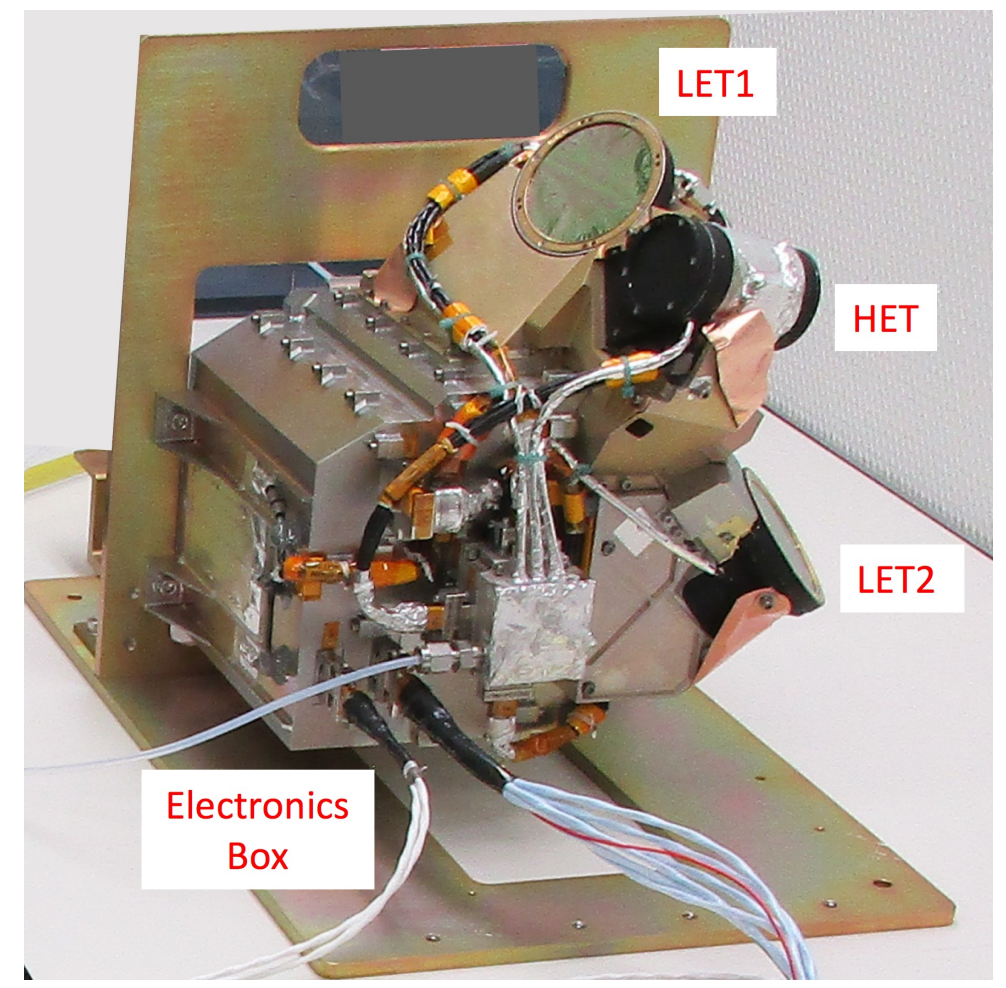

Figure 1: EPI-Hi mounted in a handling fixture during final source testing prior to delivery to the spacecraft in May 2017. For scale: the LET1 window has a diameter of $4.2 \mathrm{~cm}$.

Figure 2 shows computer images of the HET and LET1 telescopes in which sections of the telescope housings have been cut away to show inside. Each detector consists of a silicon wafer in a polyimide mount (green). The active parts of the silicon detector are shown in orange and the inactive parts in yellow. Also shown are the aluminum telescope bodies (brown and beige) and the Kapton entrance windows (light blue). Starting from its entrance aperture, LET2 houses a complement of detectors corresponding to the LET1's L0A through L3B, omitting detectors corresponding to $\mathrm{L} 2 \mathrm{~B}, \mathrm{~L} 1 \mathrm{~B}$, and $\mathrm{L} 0 \mathrm{~B}$.

The active areas of the detectors are segmented to provide coarse information about particle directions of incidence and to allow dynamic adjustment of the geometrical factor for electrons, protons, and alpha particles by selectively raising trigger thresholds on some segments. This "dynamic thresholds" technique has been used on the STEREO/LET instrument [4]. The detectors in the central stacks are closely spaced $(0.5 \mathrm{~mm}$ between facing silicon surfaces) to minimize susceptibility to backgrounds entering through the sides of the telescopes. There are larger gaps $(\sim 1 \mathrm{~cm})$ between the outer detectors (HET's H1 and H2; LET's L0, L1, and L2) to provide a sufficient flight path for particle-trajectory determination. 

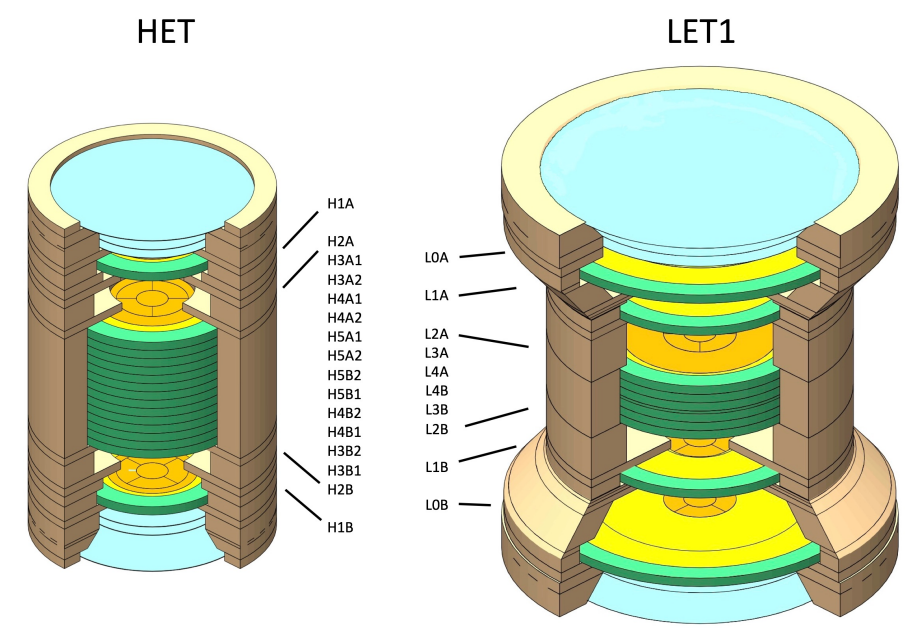

Figure 2: Drawings of HET and LET1 telescopes shown to the same scale with portions of the housings cut away to show the detectors and windows.

In order to achieve a low threshold energy for the $d E / d x-E$ measurements, the front elements of the LET1 telescope have been kept thin, with a total of $4 \mu \mathrm{m}$ of Kapton in the windows plus $12 \mu \mathrm{m}$ and $25 \mu \mathrm{m}$ in the L0 and L1 silicon detectors, respectively. This allows two-parameter measurements for protons down to $\sim 1 \mathrm{MeV}$ and to $\lesssim 2 \mathrm{MeV} /$ nuc for all elements up through $Z=28$. In LET2, the combined thickness of the windows is $16 \mu \mathrm{m}$, which is four times the thickness used in LET1. This difference, which results in a higher energy threshold for LET2 (by between $\sim 0.5$ and $\sim 1.5 \mathrm{MeV} / \mathrm{nuc}$ ), was driven by the desire to reduce the risk of detector damage due to impacts by fast dust particles orbiting the Sun in at least one LET aperture.

Except at the lowest energies, LET determines incidence directions based on the hit sectors in the L1 and L2 detectors, without a requirement for a signal in L0. Many of these trajectories will miss the active elements of $\mathrm{L} 0$, so that detector has been designed to have a uniform thickness over a significantly larger silicon area, as can be seen in the photographs in Figures 3(a) and 3(b). This enables a simple correction for the unmeasured energy loss in L0 (and in the windows).

\section{Test Results}

EPI-Hi has been tested using radioactive sources and accelerator beams. The LET windows and L0 detectors are thin enough to allow alpha particles from a ${ }^{228} \mathrm{Th}$ source (maximum energy $\sim 2.2 \mathrm{MeV} /$ nuc) to provide two-parameter $d E / d x-E$ measurements if the telescope is evacuated or filled with He gas. Figure 4(a) shows the low-energy He response track obtained in this way, demonstrating the capability to resolve ${ }^{3} \mathrm{He}$ from ${ }^{4} \mathrm{He}$ at the lowest energies. In flight, enhanced ${ }^{3} \mathrm{He} /{ }^{4} \mathrm{He}$ ratios will serve as important indicators of particle acceleration in impulsive (magnetic reconnection associated) solar energetic particle events. Tests with light isotopes at higher energies were performed by fragmenting a heavy-ion beam from the Texas A\&M University (TAMU) K500 cyclotron. These measurements are illustrated in Figure 4(b).

Element separation capabilities were tested over a wide energy range at TAMU and at the Michigan State University (MSU) National Superconducting Cyclotron Laboratory. Examples of 

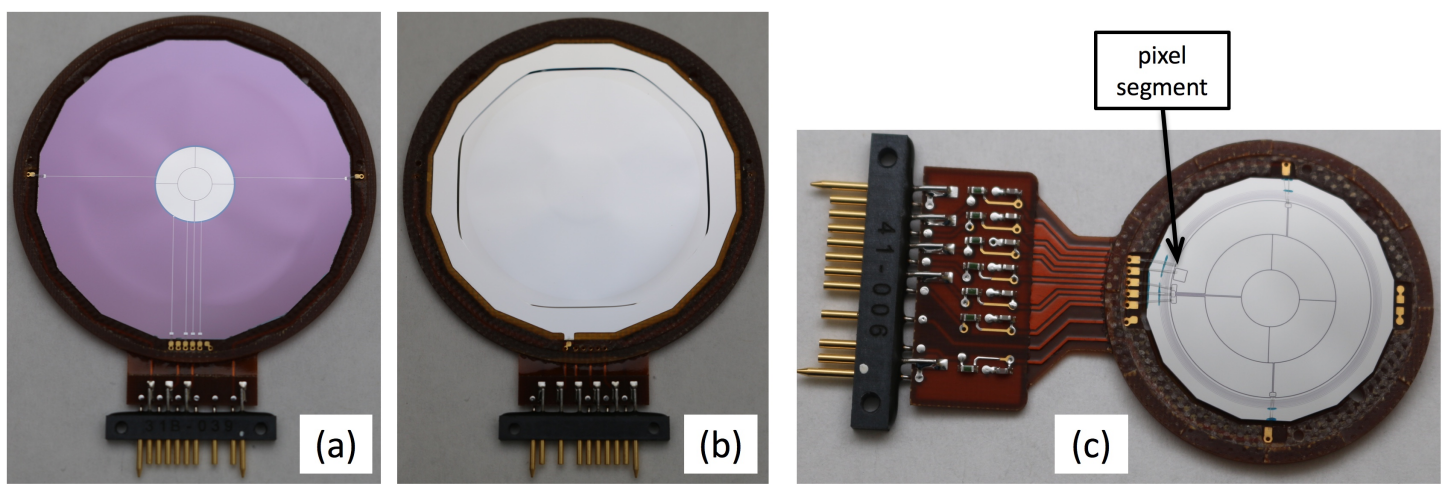

Figure 3: Detector photographs: (a) Front (junction) surface of an L0 detector. At the center are five active elements, each with an area of $0.2 \mathrm{~cm}^{2}$, arranged as a central bull's eye surrounded by four quadrants. Both the active elements and the surrounding inactive area have a thickness of $12 \mu \mathrm{m}$. (b) Back (ohmic) surface of the $\mathrm{L} 0$ detector. The $12 \mu \mathrm{m}$ membrane is supported by a thick $(500 \mu \mathrm{m})$, outer frame. This thick/thin structure is etched from a silicon-on-insulator wafer. (c) $\mathrm{H} 2$ detector, with a thickness of $1 \mathrm{~mm}$, has the same pattern of five central active elements as the L0 detector. It is surrounded by an annular "guard" element used for vetoing particles that enter or exit through the edges. A small "pixel" element with an area of $1 \mathrm{~mm}^{2}$ is used for measuring the count rate of stopping protons under the most extreme high-rate conditions. The diameter of the central five active elements on each detector is $1.13 \mathrm{~cm}$ for a combined active area of $1.0 \mathrm{~cm}^{2}$.

the track separation in LET are shown at low and intermediate energies in panels (a) and (b), respectively, of Figure 5. Tracks measured for HET (not shown) demonstrated element resolution comparable to that shown in Figure 5(b).
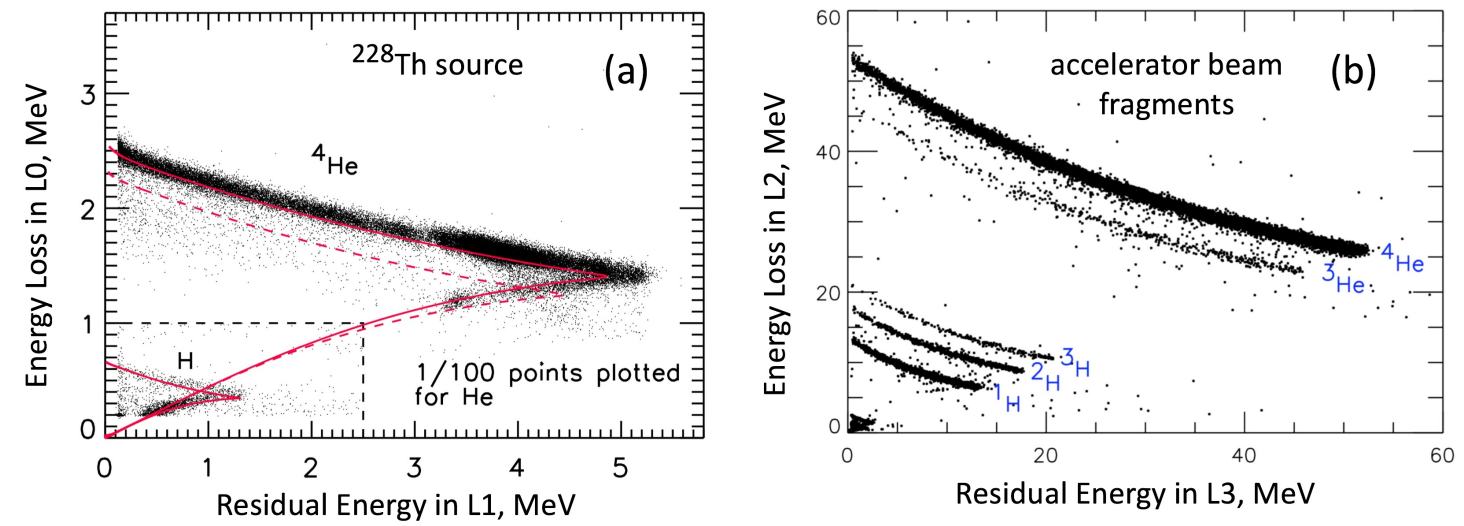

Figure 4: Measured light-isotope response tracks: (a) ${ }^{4} \mathrm{He}$ from a ${ }^{228} \mathrm{Th}$ alpha-particle source together with a small amount of ${ }^{1} \mathrm{H}$ produced as knock-on protons in a plastic mesh placed between the source and the detectors. The dashed line shows the expected location of the ${ }^{3} \mathrm{He}$ track when the detector parameters are adjusted to make the calculated ${ }^{4} \mathrm{He}$ track (upper solid line) match the measured track. (b) $\mathrm{H}$ and He isotopes produced by fragmentation of a heavy-ion beam at the Texas A\&M cyclotron. In both cases, thin, plastic mesh material was placed in front of the telescope to obtain a spread of particle energies. 

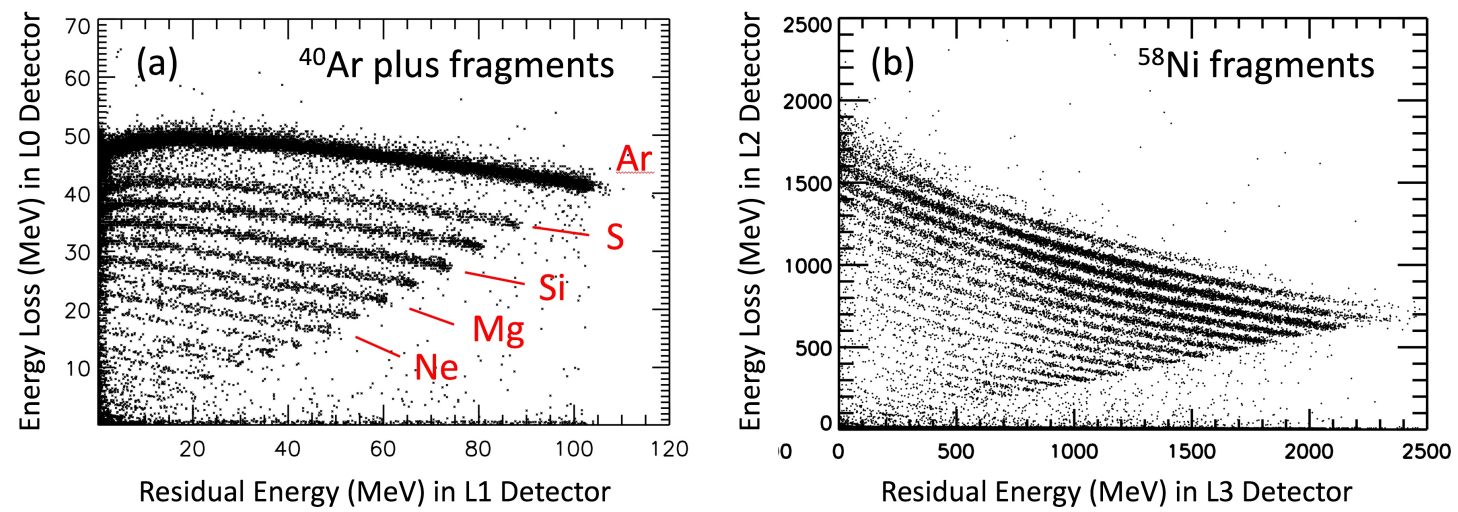

Figure 5: Energy loss $(\Delta E)$ versus residual energy $\left(E^{\prime}\right)$ plots showing element resolution. (a) LET2 measurements of low-energy ${ }^{40} \mathrm{Ar}$ and interaction products produced in $\mathrm{CH}_{2}$ target at the Texas A\&M cyclotron. The $\Delta E$ signal is from a $12 \mu \mathrm{m}$ thick L0 detector and the $E^{\prime}$ signal is from a $25 \mu \mathrm{m}$ thick L1 detector. (b) LET1 measurements of medium-energy fragments produced by interactions of a ${ }^{58} \mathrm{Ni}$ beam from the Michigan State cyclotron in a $\mathrm{H}_{2} \mathrm{O}$ target. The $\Delta E$ and $E^{\prime}$ detector thickness are $500 \mu \mathrm{m}$ (L2) and $1000 \mu \mathrm{m}$ (L3), respectively.

\section{Simulations}

The EPI-Hi response to electrons is studied using Geant4 [5] simulations, with spot checks using $\beta$-particle sources. Geant 4 is also employed to explore a parameter space for protons and heavy ions that is more extensive than could be covered with available accelerator beams. Figure 6 shows calculated geometrical factors (heavy black curves) for electrons and for protons in HET and LET1 as a function of incident energy. These calculations, which consider only particles incident from the A-end of each telescope, identify the depth to which each simulated particle penetrated in the telescope and divide up the total geometrical factor into individual "ranges", shown using thin solid lines. The shortest ranges in the four panels are: (a) L3A; (b) H2A; (c) L1A; (d) H2A. The LET electron geometrical factor (panel a) at the highest energies is due to particles that scatter out of the sides of the telescope. Such effects are much less significant in HET (panel b), which has annular "guard" segments that are used to veto a large fraction of particles exiting through the sides. Simulations of this type will be important for calculating detection efficiencies needed to derive the incident electron spectra from measured count rates.

\section{Additional Capabilities}

The EPI-Hi design enables a variety of additional capabilities that should contribute to understanding particle acceleration processes close to the Sun.

Pitch-angle distributions can be studied using information about particle incidence directions in combination with magnetic field measurements to be provided by the magnetometers that are part of the FIELDS suite [6]. For ions, up to 25 overlapping sectors are defined based on the hit segments in two position-sensitive detectors. For electrons, broad sectors are defined by the collimation provided by each telescope entrance aperture, with some broadening due to multiple scattering in the windows and the front detectors. 

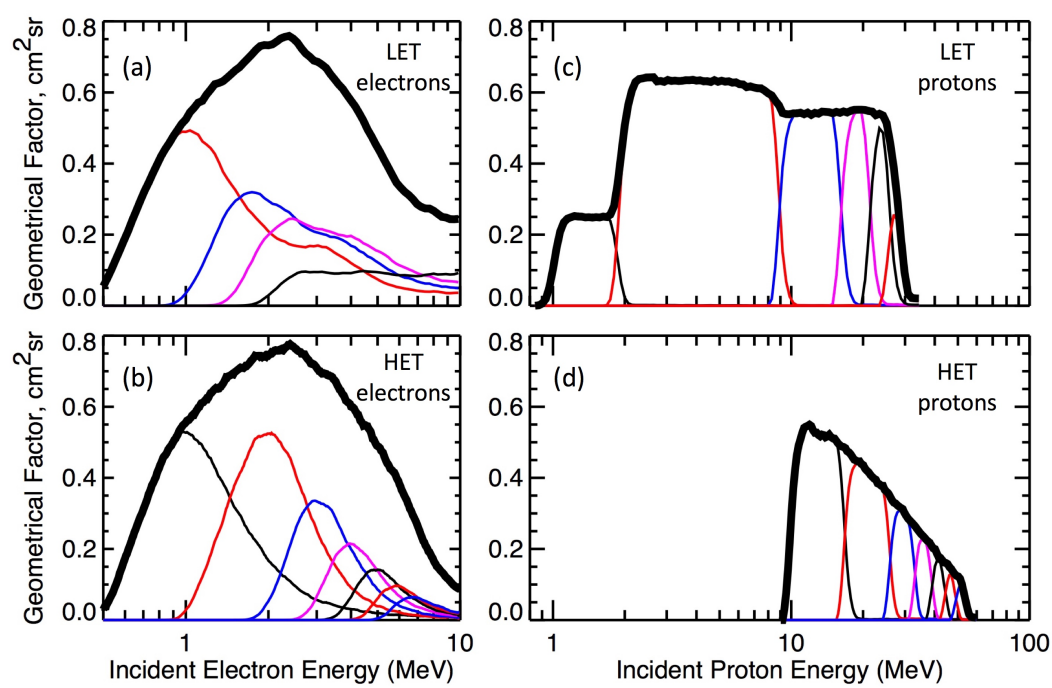

Figure 6: Geometrical factor as a function of incident energy for electrons (panels a \& b) and protons (c \& d) measured in LET1 (a \& c) and HET (b \& d). In addition to total geometrical factors, component geometrical factors for particles stopping in successive detectors in the telescopes are shown. The calculations are based on Geant4 simulations.

Besides the stopping ions that contribute to the geometrical factors shown in Figures 6(c) and (d), EPI-Hi identifies penetrating ions. Using the change in the measured $d E / d x$ value from the front to the back of a telescope, it is possible to identify major elements and calculate incident energies, provided that the $d E / d x$ increase is at least $\sim 10 \%$.

Solar energetic particle event intensities close to the Sun can greatly exceed those seen in the same event near Earth because of expansion in space $\left(\sim r^{2}\right)$ and time. If a large event occurs when PSP is close to perihelion, count rates in the $0.2 \mathrm{~cm}^{2}$ segments of the EPI-Hi detectors can be high enough to saturate the front-end electronics. In order to obtain some information about proton intensities in such extreme events, small pixel segments $\left(\sim 1 \mathrm{~mm}^{2}\right.$ area) are instrumented on some of the thick HET and LET detectors (see Fig. 3(c)). By setting pixel thresholds at a significant fraction of the energy that would be deposited by a proton just stopping in $1 \mathrm{~mm}$ of $\mathrm{Si}$ $(\sim 12 \mathrm{MeV})$, coarse information about the proton spectrum can be obtained from pixel count rates, since different amounts of material must be penetrated to reach the different instrumented pixels. It should be possible to calibrate these measurements in space by comparing with spectra measured in the full telescope at intensities somewhat below where saturation sets in.

Nuclear interactions in the solar atmosphere produce neutrons and gamma rays. These neutral radiations can provide important information about particle acceleration deep in the corona. Resource limitations prevented inclusion of a dedicated neutron and gamma-ray spectrometer in the PSP payload. By taking advantage of signals from the inner detector elements in the telescopes while vetoing events that also trigger surrounding outer segments, EPI-Hi should be able to make exploratory measurements of these neutrals. The flight software has been designed to analyze such events. Laboratory measurements have been carried out with neutron and gamma-ray sources to aid in interpreting these measurements, and Geant4 simulations are planned. 
Measurements with the STEREO/LET instruments [7] led to the serendipitous discovery of energetic neutral hydrogen atoms in a solar energetic particle event. Close to the Sun, EPI-Hi's fields of view will include the upper part of the corona where such neutrals might be produced as protons accelerated deeper in the corona get neutralized by attaching an electron from the ambient gas. If these neutrals escape before being reionized, EPI-Hi should be able to identify them as protons coming directly from the point of neutralization. Under some circumstances they may be detectable before being overwhelmed by a large population of accelerated protons. Correlated measurements of the two populations may provide new insights into the details of proton acceleration and escape.

\section{Conclusion.}

The EPI-Hi instrument on the Parker Solar Probe mission will explore particle acceleration and transport in regions of the heliosphere much closer to the Sun than has previously been possible. These unique measurements, together with those by EPI-Lo [3] and the European Solar Orbiter mission [8], have the potential to greatly advance our understanding of the radiation environment produced by the Sun.

\section{Acknowledgments}

We thank C. Anderson and D. Miles for administrative support at Caltech, Micron Semiconductor, Ltd. for developing the EPI-Hi detectors, D. Aalami of Space Instruments for developing the bias supply, and the cyclotron laboratories at Michigan State University, Texas A\&M University, and the Lawrence Berkeley National Laboratory for providing calibration beams. Part of the research was carried out at the Jet Propulsion Laboratory, California Institute of Technology, with support from the National Aeronautics and Space Administration.

\section{References}

[1] G. Wibberenz \& H. V. Cane, Multi-Spacecraft Observations of Solar Flare Particles in the Inner Heliosphere, Astroph. J., 650, 1199, 2006.

[2] N. J. Fox et al., The Solar Probe Plus Mission: Humanity's First Visit to Our Star, Space Sci. Rev., 204, 7, 2016.

[3] D. J. McComas et al., Integrated Science Investigation of the Sun (ISIS): Design of the Energetic Particle Investigation, Space Sci. Rev., 204, 187, 2016.

[4] R. A. Mewaldt et al., The Low-Energy Telescope (LET) and SEP Central Electronics for the STEREO Mission, Space Sci. Rev., 136, 285, 2008.

[5] J. Allison et al., Recent Developments in Geant4, Nucl. Instr. \& Meth. in Phys. Res. A, 835, 186, 2016.

[6] S. D. Bale et al., The FIELDS Instrument Suite for Solar Probe Plus, Space Sci. Rev., 204, 49, 2016.

[7] R. A. Mewaldt et al., STEREO Observations of Energetic Neutral Atoms during the 2006 December 5 Solar Flare, Astroph. J., 693, L11, 2009.

[8] R. Gómez-Herrero et al., The Solar Orbiter Mission: an Energetic Particle Perspective, eConf C16-09-04.3, 2016 [arXiv: 1701.04057v1]. 